\title{
Characterization and Modeling of Coarsening Mechanisms in Supported Nanoparticle Ensemble.
}

Dmitri N. Zakharov ${ }^{1 *}$, Alexei Tkachenko ${ }^{1}$, Xiaohui Qu ${ }^{1}$, Hong Wang ${ }^{2}$, Yuewei Lin ${ }^{3}$, James P. Horwath ${ }^{4}$, Shinjae $\mathrm{Yoo}^{3}$, and Eric A. Stach ${ }^{4}$

1. Center for Functional Nanomaterials, Brookhaven National Laboratory, Upton, NY 11973, USA

2. Department of Applied Mathematics and Statistics, Stony Brook University, Stony Brook, NY 11794, USA

3. Computational Science Initiative, Brookhaven National Laboratory, Upton, NY 11973, USA

4. Department of Materials Science and Engineering, University of Pennsylvania, Philadelphia, PA 19104, USA

* Corresponding author: dzakharov@bnl.gov

Nanoparticle synthesis and assembly are vital elements of the nanotechnological revolution. They impact multiple applications, ranging from nanocatalysis to optoelectronics and structural reinforcement. Environmental aberration-corrected transmission electron microscopy combined with MEMS-based heating holders and direct electron detectors allows for direct detailed observation of nanoparticle coarsening with high spatial and temporal resolution. Here we report on evolution mechanisms of $\mathrm{Au}$ particles supported on $\mathrm{SiN}$ thin films during annealing at $950^{\circ} \mathrm{C}$ for over 120 minutes (Figure 1a). The study is done in situ using an Environmental FEI Titan 80-300 (S)TEM operated at 300kV, equipped with a DENS Solutions heating holder and Gatan direct electron detector capable of acquiring streams of 1920 x 1792 pixels images at 400 frames per second. This results in streaming data rate of approximately $3 \mathrm{~GB} / \mathrm{s}$. An automatic particle indexing procedure [1] was applied to TEM images to extract particles sizes and positions for progressive time frames (Figure 1b).

Quantitative analysis of extracted data allowed us to separate and characterize different mechanisms leading to particle coarsening [2,3]. In particular, the dominant mechanism that leads to increased average size with time is the coalescence associated with effective diffusion and "collisions" of particles. This mechanism is also responsible to decrease of net number of particles (Figure 1c,d). The other mechanism is due to the sublimation of gold atoms from particles and support leading to slow net loss of gold in the field of view (Figure 2a). The two processes differ by almost the order of magnitude: coalescence gives an average rate on the scale of $1 \mathrm{~nm}^{3} / \mathrm{min}$, while sublimation gives negative rate around $0.2 \mathrm{~nm}^{3} / \mathrm{min}$ (Figure 2a,c). Remarkably, the classical Ostwald ripening behavior is completely overshadowed by the strong variation of the sublimation rate even for particles of similar sizes. While the average rate is only weakly dependent on the particle volume (black solid line in the insert in Figure 2a), its dispersion is always significant and shows a strong increase with a particle volume (Figure 2b). We associate this variation with effects associated with the heterogeneity of local particle environment.

This data provides valuable insight into nanoparticle coarsening processes and enriches our understanding of these mechanisms, including revision of the classical Ostwald ripening picture. Furthermore, the observed prominent role played by the local particle environment opens an opportunity to manipulate this environment, and thus, optimize nanoparticle manufacturing for specific applications $[4]$. 
References:

[1] RC Gonzalez, RE Woods in "Digital Image Processing”, (Pearson, 2017)

[2] M Zinke-Allmang, Thin Solid Films 346 (1999), p.1.

[3] WC Yang, M Zeman, H Ade, R Nemanich, Physical review letters 90 (2003), p.136102.

[4] This research used resources of the Center for Functional Nanomaterials, which is a U.S. DOE Office of Science Facility, at Brookhaven National Laboratory under Contract No. DE-SC0012704. JPH and EAS acknowledge additional support from the National Science Foundation, Division of Materials Research through Award \#1809398.

(a) $50 \mathrm{~nm}$

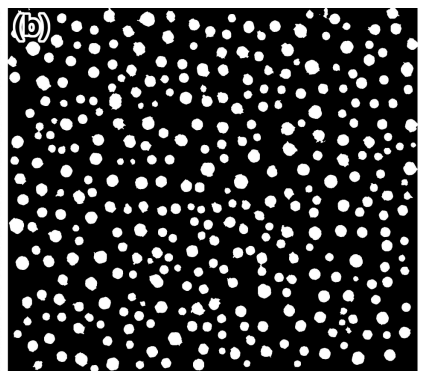

(d)

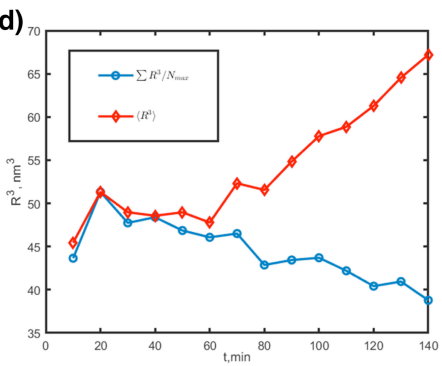

Figure 1. (a) TEM image frame extracted from a data set acquired during annealing of Au nanoparticles supported on an amorphous SiN film as a column pressure of $10^{-7}$ Torr and $950^{\circ} \mathrm{C}$ for over 120 minutes. (b) Threshold image of the frame in (a) generated by an automatic particle indexing procedure. (c) Number of particles per unit area versus time. (d) Normalized total volume of particles in the field of view (blue) and average particle volume (red) versus annealing time.
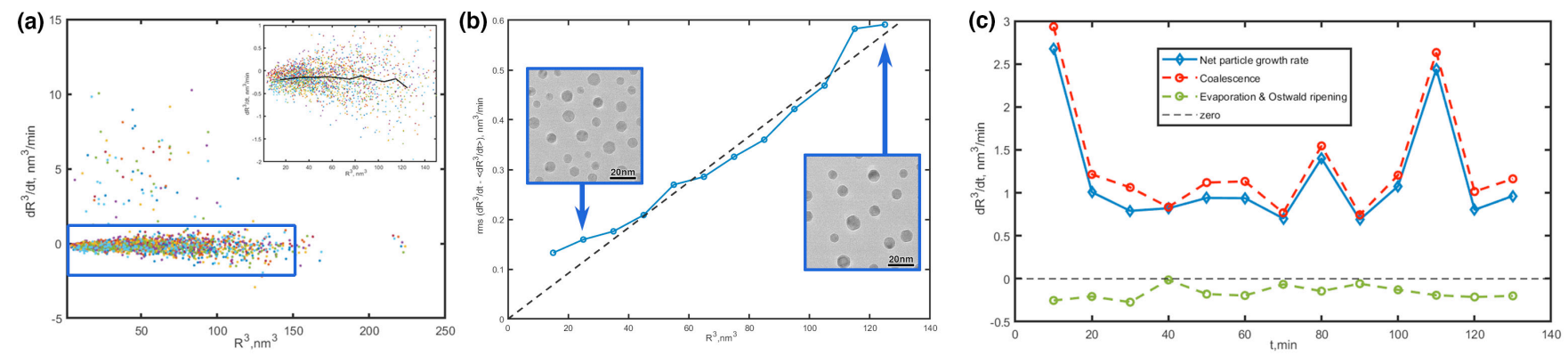

Figure 2. (a) Particle growth rate versus volume. Inset: small rate data dominated by sublimation and Ostwald ripening. (b) Dispersion of growth rate versus particle volume. (c) Contributions of different coarsening mechanisms towards net particle growth rate versus time. 